\title{
Extranodal natural killer/T-cell lymphoma presenting as cavernous sinus syndrome
}

\author{
HONG-CHENG MAI, DAN-XIA CHEN, DAN LU and YU-SHENG ZHANG
}

\begin{abstract}
Department of Neurology, The First Affiliated Hospital of Jinan University, Guangzhou, Guangdong 510632, P.R. China
\end{abstract}
Received November 17, 2016; Accepted January 10, 2017

DOI: $10.3892 / \mathrm{mco} .2017 .1190$

\begin{abstract}
Extranodal natural killer (NK)/T-cell lymphomas are rare, highly aggressive tumors of the central nervous system (CNS) that commonly affect the nasal cavity or paranasal sinuses. NK/T-cell lymphomas are associated with a poor prognosis, as they exhibit a wide range of unique clinical presentations and neuroimaging findings that are currently difficult to detect. Pathological examination is considered to be more reliable for defining the pathological entity of NK/T-cell lymphoma. Early testing for fluorodeoxyglucose (FDG)-positron emission tomography combined with computed tomography may improve the evaluation of lymphomas, which have been known to display avid ${ }^{18} \mathrm{FDG}$ uptake. The authors herein report a case of NK/T-cell lymphoma presenting with cavernous sinus syndrome involving the CNS via direct extension from the nose. NK/T-cell lymphomas are very aggressive, with an unfavorable clinical outcome, and the optimal therapy has not yet been clearly defined.
\end{abstract}

\section{Introduction}

Primary central nervous system (CNS) lymphomas originating from the brain, leptomeninges, spinal cord, or eyes are rare tumors, accounting for $<2 \%$ of all cerebral neoplasms (1). Local mass effect may result in ocular involvement, or focal lesion of the cranial nerve roots. As the blood-brain barrier may impair drug delivery, the available treatments for primary natural killer (NK)/T-cell lymphomas are limited. Early possible diagnostic neuroimaging is therefore important. Positive cerebrospinal fluid cytology or brain biopsy results may provide definitive proof of primary NK/T-cell lymphomas. Modern imaging technology has demonstrated that these lesions have a characteristic appearance on neuroimaging. Typical NK/T-cell lymphomas appear as hypointense lesions

Correspondence to: Dr Yu-Sheng Zhang, Department of Neurology, The First Affiliated Hospital of Jinan University, 603 Huangpu West Avenue, Guangzhou, Guangdong 510632, P.R. China

E-mail: zhangys@jnu.edu.cn

Key words: central nervous system lymphoma, cavernous sinus, natural killer/T-cell lymphoma, immunohistochemistry, biopsy on long TR-weighted magnetic resonance imaging (MRI) of the brain. Lesions exhibiting enhancement on computed tomography (CT) scans following contrast administration are considered to be deposits of lymphoma cells (2). In particular, increased glycose metabolism in areas of the brain on positron emission tomography (PET)-CT is an important distinguishing characteristic of these lesions (3).

The majority of extranodal NK/T-cell lymphomas are pathological entities distinct from B- or T-cell lymphomas. Primary NK/T-cell lymphomas are more rare and, although generally rare, they are more commonly encountered in individuals of Asian descent. On immunohistochemical examination, lesions of extranodal NK/T-cell lymphomas often consist of CD2-positive, surface CD3-negative, cytoplasmic CD3-positive and CD57-positive cells $(4,5)$. As extranodal NK/T-cell lymphomas often affect the nasal cavity, they frequently present in midline facial structures accompanied with nasal obstruction. However, NK/T-cell lymphomas causing cavernous sinus syndrome via direct destruction of the cavernous sinus and cranial nerves are rarely reported.

\section{Case report}

A 39-year-old man in good general health was first admitted to The First Affiliated Hospital of Jinan University (Guangzhou, China) on November 17, 2013. The patient presented with sudden onset of epistaxis and was admitted to the ENT department due to diagnosis of nasal polyps by nasal endoscopy. On November 27 (3 days after nasal surgery), the patient developed fever, headache and diplopia associated with pain in the right orbital region. Due to worsening of the symptoms, he was referred to the Neurology Department. On neurological examination, there was paralysis of the right III and VI cranial nerves, with ptosis and bilateral eyelid edema. The right pupil measured $4.0 \mathrm{~mm}$ and did not constrict to direct light; the left pupil measured $3 \mathrm{~mm}$ and the pupillary light reflex was normal. A peanut-sized nodule was found in right medial canthus. Sensation in the area of right trigeminal nerve was lost. The patient's diplopia was associated with ophthalmoplegia and third and sixth cranial nerve palsy on the right side. The findings of the general examination and chest radiograph were normal. The result of the laboratory examinations were as follows: The erythrocyte sedimentation rate was $50 \mathrm{~mm} / \mathrm{h}$ and the high-sensitivity C-reactive protein was $91.79 \mathrm{mg} / 1$. 

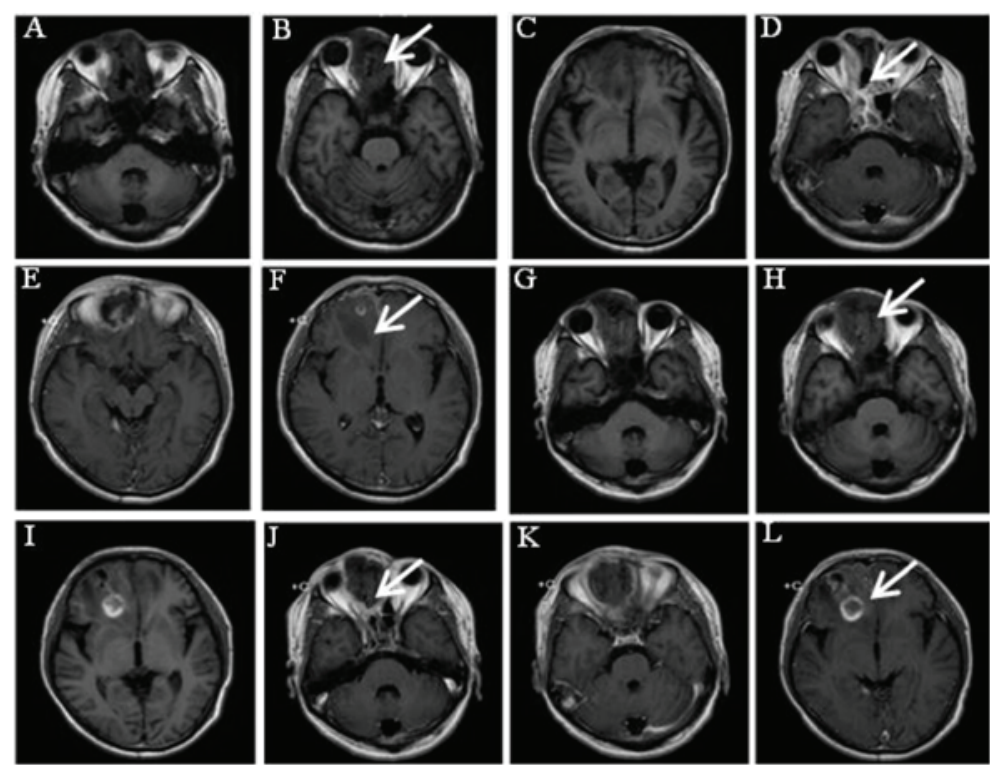

Figure 1. Brain magnetic resonance imaging (MRI). (A-F) Initial MRI evaluation revealed focal abnormal signal lesions (white arrows). These lesions were hyperintense on T1-weighted images, showing a tumor involving the bilateral maxillary sinus, sphenoid sinus and cavernous sinus. Images of right frontal lobe on axial T1-weighted images appeared edematous and exhibited circular enhancement (white arrow). (G-L) Assessment 1 month after the initial evaluation revealed rapid disease progression. (A-C and G-I) Axial T1-weighted images. (D-F and J-L) Enhanced axial T1-weighted images.

Diagnostic lumbar spinal puncture on December 19 revealed a high pressure of $195 \mathrm{mmH}_{2} 0$. The nasal secretions culture was positive for Enterococcus avium and the cerebrospinal fluid culture was positive for Staphylococcus aureus. A naso-antral CT scan revealed paranasal sinusitis, which was attributed to inflammation following bilateral nasal polypectomy on December 7. A brain MRI on December 16 showed an area of abnormal signal in the right frontal lobe and cranium (Fig. 1). Compared to the former MRI, moderate annular enhancement of the lesion was observed, exhibiting T2-weighted hyperintensity and T1-weighted hypointensity in the right frontal lobe. The maxillary sinus bilaterally, the sphenoid sinus and the frontal sinus exhibited long $\mathrm{T} 1$ and $\mathrm{T} 2$ signal. The mucosa of the sinus wall exhibited an annular enhancement sign on enhancement scan. Repeat MRI after 1 month showed progression of the lesions in the frontal lobe (Fig. 1). The patient improved rapidly within 7 days after the initiation of ceftriaxone sodium and glucocorticoid treatment. The glycose metabolism was found to be increased in the right side of the nose, cavernous sinus and sphenoid sinus on PET-CT examination (Fig. 2). Following pathological examination, the patient was diagnosed with lymphoma metastatic to the lymph nodes.

Further investigation identified the mass from the nasal cavity as extranodal NK/T-cell lymphoma (Fig. 3). Re-examination of the cranial nerves revealed partial recovery of the III and VI nerves. The symptoms of fever, headache and diplopia associated with pain in the right orbital region subsided significantly following antibiotic and glucocorticoid treatment. However, similar severe symptoms suddenly developed, accompanied by muscle twitching in the limbs and coma. The right eye developed severe erythema and edema, with fever and headache. The patient developed kidney and heart failure and succumbed to the disease 4 months after the initial diagnosis.

\section{Discussion}

Cavernous sinus syndrome was first described by Foix in 1921 (also referred to as Foix's syndrome) and is most commonly caused by vascular disease, tumors and the Tolosa-Hunt syndrome (6). An investigation of 102 cavernous sinus syndrome patients revealed that $69 \%$ of the cases resulted from tumors, $19 \%$ from vascular diseases and $12 \%$ from inflammation. Another etiological study including 151 cavernous sinus syndrome patients also reported similar results, namely that the major causative factors are tumors (7). Primary brain tumors, such as meningiomas, as well as metastatic brain tumors may lead to cavernous sinus syndrome. Non-Hodgkin lymphoma (NHL) comprises a group of aggressive lymphoproliferative malignancies originating from lymphoid tissue that usually invade the CNS (8). However, there is currently a lack of reports on cavernous sinus syndrome from NHL. Extranodal NK/T-cell lymphoma presenting with cavernous sinus is more rare in clinical practice, with very few cases reported to date.

Extranodal NK/T-cell lymphomas are uncommon, highly aggressive malignant lymphomas, which exhibit a strong association with episomal Epstein-Barr virus (9) and have a higher incidence in Asia, Mexico and South America (10). The nasal cavity is the most common site of involvement, as was the case in our patient, who presented with sudden onset of epistaxis; however, other sites were also affected, including the paranasal sinuses, pharynx and CNS, where destructive lesions may present with nasal obstruction, facial pain, or edema. The case reported herein is significant. The cavernous sinus is a pentahedron that contains cranial nerves b, c, $\mathrm{d}$ and e and the carotid artery. When the cavernous sinus is affected by traumatic or infectious lesions, vascular or neoplastic processes, the presenting signs and symptoms may include orbital or retroorbital pain, diplopia and Horner's syndrome due to the 

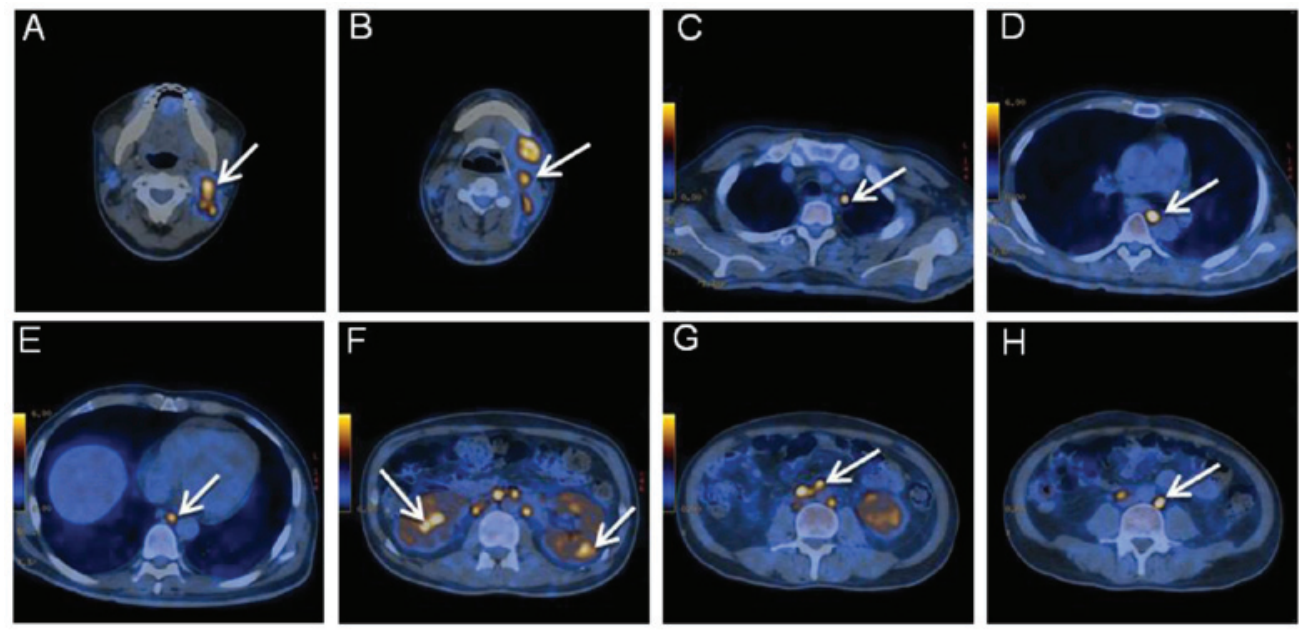

Figure 2. Positron emission tomography/computed tomography image of extranodal natural killer (NK)/T-cell lymphoma. (A and B) Lesions of NK/T-cell lymphoma in the cavernous sinus (arrows). (C-E) Relapse of nasal NK/T-cell lymphoma in the mediastinum (arrows). (F) Disseminated NK-cell lymphoma showing diffuse uptake of fluorodeoxyglucose in the kidneys (arrows). (G-H) Abdominal NK/T-cell lymphoma exhibiting increased glucose metabolism (arrows).
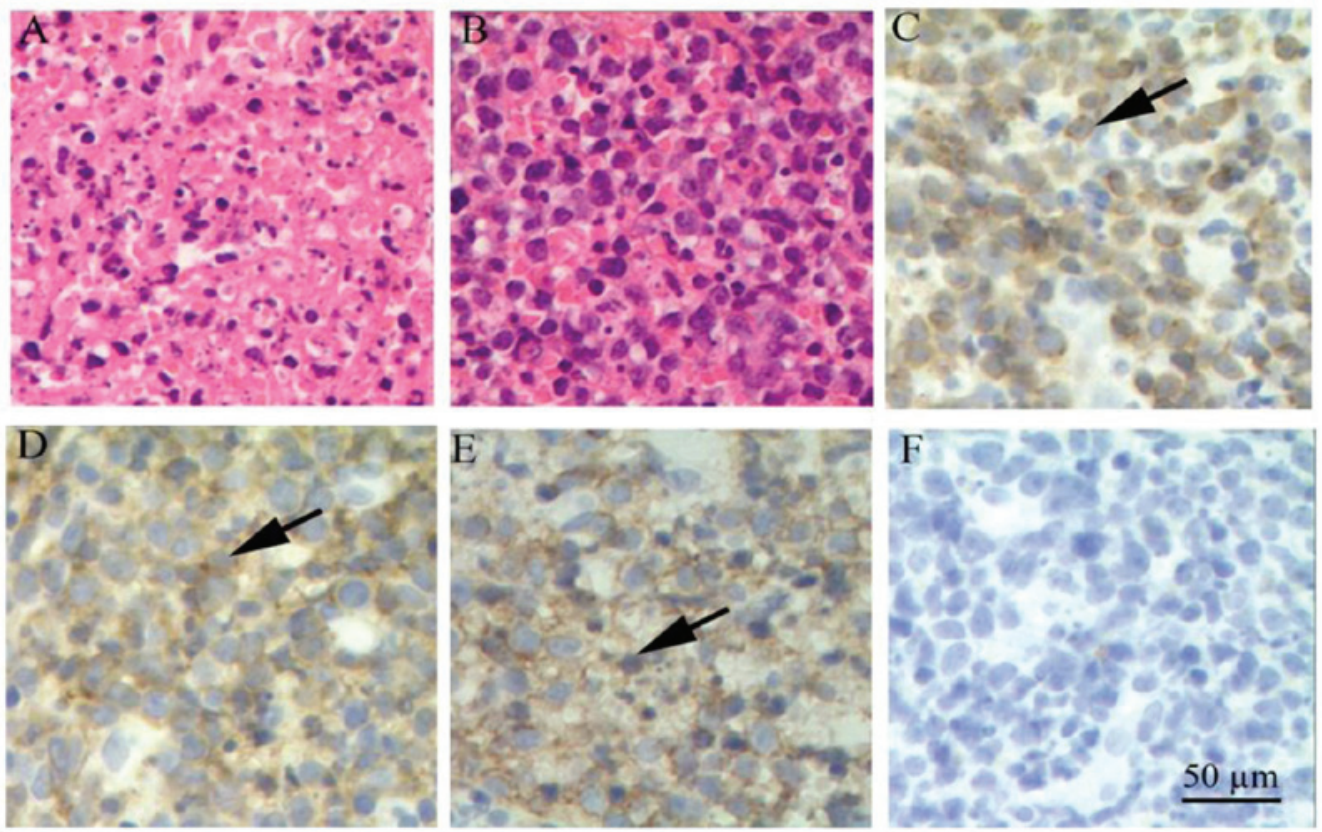

Figure 3. On histopathological examination, lymphoma cells infiltrating the nasal cavity were observed. (A and B) Biopsy of the nasal cavity mass (hematoxylin and eosin staining). The infiltrating cells were small- to medium-sized lymphocytes. Immunohistochemical staining of the tumor cells showed (C) positivity for the T-cell marker CD3 (arrow). Further marker studies showed positivity for (D) CD45RO (leukocyte common antigen) and (E) CD57 (respective arrows), indicating extranodal, nasal-type natural killer/T-cell lymphoma. (F) The tumor cells were negative for the B-cell marker CD20. Original magnification, x400.

involvement of one or more related eye muscles and nerves or branches of the trigeminal nerve.

Histologically, NK/T-cell lymphomas typically express both T-cell and NK-cell immunophenotypes and NK and T cells share a common ontogenesis and express cytoplasmic CD3 antigen (11). In addition, NK cells consistently express CD56 and CD57, which are considered as NK-associated antigens (12).

In the present case, the patient was originally admitted to the hospital with fever of unknown origin and epistaxis, which may be easily misdiagnosed as a common cold. However, the typical symptoms or signs of cavernous sinus syndrome, together with abnormal solid lesions on MRI, should raise concerns in the attending doctors, as early findings may affect later diagnostic testing and treatment. PET-CT is useful for staging and is recommended in the clinical setting when more accurate evaluation may alter management. PET-CT has a higher sensitivity in aggressive extranodal NK/T-cell lymphomas when compared with CT alone and is widely recognized as the most sensitive and specific imaging for patients with NHL, particularly extranodal NK/T-cell lymphomas (13). When PET/CT is unavailable, CT or MRI may be considered.

As extranodal NK/T cell lymphoma is one of the most difficult subtypes to treat and is associated with a poor 
prognosis, current treatment strategies are adopted from retrospective analyses and have not been clear defined. Reviewing the treatment strategies, these currently include radiotherapy, intensive chemotherapy and surgery for patients with early-stage disease. For remission of early-stage lymphomas, the role of hematopoietic stem cell transplantation is not indicated for prospective evaluation. Radiotherapy together with chemotherapy is the standard approach for localized disease. Systemic chemotherapy consisting of CNS prophylaxis for this patient remains the mainstay of treatment (14). The indication for patients with CNS involvement is early recurrence with rapid progression, which was the case in our patient. However, no treatment has been found to be sufficiently effective, and this malignancy manifests early signs of aggression. It was previously reported that the median survival time of nasal-type extranodal NK/T-cell lymphoma is 2 years in patients responding poorly to standard therapies (15). Accompanied by systemic progression of this disease, death often ensues within 1 year of diagnosis.

In summary, we herein present the case of a patient with cavernous sinus syndrome, in which an autopsy confirmed extranodal NK/T-cell lymphoma with multiple metastases. MRI and PET-CT imaging should provide sufficient evidence for the diagnosis of early-stage extranodal NK/T-cell lymphoma and early treatment of aggressive disease may significantly improve outcome. Clinicians should apply the findings from clinical studies to targeted therapy.

\section{Acknowledgements}

The present study was supported by grants from the National Natural Science Foundation of China (nos. 81171084 and 81671167), the Science and Technology Program of Guangzhou (nos. 155700029 and 1561000289), the Natural Science Foundation of Guangdong Province (no. 2014A030313384) and the Medical Scientific Research Foundation of Guangdong Province (no. A2014381), P.R. China.

\section{References}

1. Liu JK, Sayama C, Chin SS and Couldwell WT: Extranodal NK/T-cell lymphoma presenting as a pituitary mass. Case report and review of the literature. J Neurosurg 107: 660-665, 2007.
2. Kwong YL: Natural killer-cell malignancies: Diagnosis and treatment. Leukemia 19: 2186-2194, 2005.

3. Khong PL, Pang CB, Liang R, Kwong YL and Au WY: Fluorine-18 fluorodeoxyglucose positron emission tomography in mature T-cell and natural killer cell malignancies. Ann Hematol 87: 613-621, 2008.

4. Sitthinamsuwan P, Pongpruttipan T, Chularojmontri L, Pattanaprichakul P, Khuhapinant A and Sukpanichnant S: Extranodal NK/T cell lymphoma, nasal type, presenting with primary cutaneous lesion mimicking granulomatous panniculitis: A case report and review of literature. J Med Assoc Thai 93: 1001-1007, 2010.

5. Sun JC and Lanier LL: NK cell development, homeostasis and function: Parallels with $\mathrm{CD} 8^{+} \mathrm{T}$ cells. Nat Rev Immunol 11: 645-657, 2011.

6. Chen SM, Chang CN, Wei KC, Jung SM and Chuang CC: Sellar lymphoma mimicking sphenoid infection presenting with cavernous sinus syndrome. J Clin Neurosci 15: 1148-1151, 2008.

7. Keane JR: Cavernous sinus syndrome. Analysis of 151 cases. Arch Neurol 53: 967-971, 1996.

8. Jahnke K, Thiel E, Martus P, Schwartz S and Korfel A: Retrospective study of prognostic factors in non-Hodgkin lymphoma secondarily involving the central nervous system. Ann Hematol 85: 45-50, 2006.

9. Li Z, Xia Y, Feng LN, Chen JR, Li HM, Cui J, Cai QQ, Sim KS Nairismägi ML, Laurensia Y, et al: Genetic risk of extranodal natural killer T-cell lymphoma: A genome-wide association study. Lancet Oncol 17: 1240-1247, 2016.

10. Kwong YL, Kim WS, Lim ST, Kim SJ, Tang T, Tse E, Leung AY and Chim CS: SMILE for natural killer/T-cell lymphoma: Analysis of safety and efficacy from the Asia lymphoma study group. Blood 120: 2973-2980, 2012.

11. Chan JK, Tsang WY and Ng CS: Clarification of CD3 immunoreactivity in nasal T/natural killer cell lymphomas: The neoplastic cells are often CD3 epsilon+. Blood 87: 839-841, 1996.

12. Sabattini E, Bacci F, Sagramoso C and Pileri SA: WHO classification of tumours of haematopoietic and lymphoid tissues in 2008: An overview. Pathologica 102: 83-87, 2010.

13. Kasenda B, Haug V, Schorb E, Fritsch K, Finke J, Mix M, Hader C, Weber WA, Illerhaus G and Meyer PT: 18F-FDG PET is an independent outcome predictor in primary central nervous system lymphoma. J Nucl Med 54: 184-191, 2013.

14. Tse E and Kwong YL: How I treat NK/T-cell lymphomas. Blood 121: 4997-5005, 2013.

15. Lee J, Suh C, Park YH, Ko YH, Bang SM, Lee JH, Lee DH, Huh J, Oh SY, Kwon HC, et al: Extranodal natural killer T-cell lymphoma, nasal-type: A prognostic model from a retrospective multicenter study. J Clin Oncol 24: 612-618, 2006. 